\title{
Anycast Routing in Delay Tolerant Networks
}

\author{
Yili Gong†, Yongqiang Xiong, Qian Zhang, Zhensheng Zhang, Wenjie Wang, Zhiwei Xu \\ gongyili@ict.ac.cn, yqx@microsoft.com, qianzh@cs.ust.hk, \\ zzhang@ieee.org, wenjiew@eecs.umich.edu and zxu@ict.ac.cn
}

\begin{abstract}
Anycast routing is very useful for many applications such as resource discovery in Delay Tolerant Networks (DTNs). In this paper, based on a new DTN model, we first analyze the anycast semantics for DTNs. Then we present a novel metric named EMDDA (Expected Multi-Destination Delay for Anycast) and a corresponding routing algorithm for anycast routing in DTNs. Extensive simulation results show that the proposed EMDDA routing scheme can effectively improve the efficiency of anycast routing in DTNs. It outperforms another algorithm, Minimum Expected Delay (MED) algorithm, by $11.3 \%$ on average in term of routing delays and by $19.2 \%$ in term of average max queue length.
\end{abstract}

\section{INTRODUCTION}

Delay Tolerant Networks (DTNs), as a class of useful but challenging networks, are receiving more and more attention. In such networks, no end-to-end contemporaneous path is guaranteed between any two nodes and message ${ }^{1}$ delivery can be fulfilled by leveraging nodes' movement. In this paper, we study the problem of anycasting in DTNs as more and more potential DTN applications need support of anycasting.

Anycast is a service that allows a node to send a message to at least one, and preferably only one, of the members in a group. The idea behind anycast is that a client wants to send packets to any one of several possible servers offering a particular service or application but does not really care any specific one. Anycast can be used to implement resource discovery mechanisms which are powerful building blocks for many distributed systems, including file sharing etc.

Anycast in DTNs means that a node wants to send a message to any one of a destination group and intermediate nodes help to deliver the message by leveraging their mobility when no contemporaneous path exists between the sender node and any node of the destination group. A typical scenario, e.g. Strawberry fair[1], shown in Fig. 1, is in a park, people cluster to watch some musical performances and they want to share and search music files at the same time. People in different clusters may be disconnected while people or cars moving between clusters can act as carriers to deliver messages. Anycast can be used to find a person who owns a certain file. Moreover, DTN anycast can be used in a disaster rescue field, in which people may want to find a doctor or a fireman without knowing their IDs or accurate locations.

$\dagger$ This work was done while Yili Gong and Wenjie Wang were visiting students at Microsoft Research Asia.

${ }^{1}$ In this paper, we use term message to represent all kinds of delivered information, including quests, replies, and files, etc and use message and packet interchangeably.
Thus efficient anycast service is important for supporting these applications in DTNs.

Though anycast in the Internet and mobile ad hoc networks has been studied extensively in the past, due to the unpredictability of network connectivity and delay, and limited storage capacity, anycast in DTNs is a quite unique and challenging problem. It requires both re-definition of anycast semantics and new routing algorithms. Moreover, in unicast in DTNs, the destination of a message is determined when it is generated, while in anycast, the destination can be any one of a group of nodes and during routing, both the path to a group member and the destination can change dynamically according to current mobile device movement situation.

In this paper, we define three semantics models of anycast in DTNs, namely CM (Current Membership), TIM (Temporal Interval Membership) and TPM (Temporal Point Membership Model), which unambiguously define the intended receivers of a message in the anycast routing. Based on the model, we also propose a novel routing metric named EMDDA (Expected Multi-Destination Delay for Anycast) which utilizes the uncontrolled random moving characteristic of mobile devices. We use a probabilistic approach that characterizes the practical average delay more accurately and develop a corresponding algorithm. The performance of the algorithm is analyzed and compared by simulation which demonstrates the effectiveness and efficiency of the proposed metric. Our simulations show that the EMDDA routing scheme can effectively improve the efficiency of anycast routing in DTNs. It achieves $11.3 \%$ lower average routing delays and $19.2 \%$ lower average max queue length compared to another algorithm, Minimum Expected Delay (MED)[2].

To the best of our knowledge, this paper is the first to systematically address the anycast routing problem in DTNs and propose the corresponding anycast routing scheme.

The rest of this paper is organized as follows. We first review related research in Section II. Section III presents our analysis of anycast in DTN and our scheme for it. The simulation and performance evaluation are discussed in Section IV. We conclude the paper with Section V.

\section{RELATED WORK}

There is a rich literature on anycast routing in the Internet and mobile ad hoc networks [3][4][5]. But these approaches can not be applicable to DTNs since it can not be assumed the connectivity is guaranteed as that in Internet, and the uncertainty of both the path to a destination group member 




Fig. 1. A typical scenario of DTNs. People clusters and cars, buses or other people moving among the clusters can act as carriers to deliver messages.

and the destination of the anycast message during the routing makes the problem more challenging.

DTN routing attracts a large amount of efforts in the research community. Vahdat and Becker firstly propose exploiting device mobility to facilitate data routing [6]. They show that with unlimited node storage capacity and time, the flooding algorithm guarantees delivery of messages. Tan et al in [7] present a routing framework named SEPR in partially connected networks. By guiding message flow to expected shortest path nodes, their approach reduces unnecessary message copies and increases the message delivering rate.

Zhao et al in [8] exploit non-randomness in a node's proactive movement to deliver messages in order to improve data delivery performance in a disconnected network. In our paper we assume that we know the mobility pattern of mobile devices but can't control it. Zhao et al also propose new semantic models for DTN multicast and develop several multicast routing algorithms with different strategies [9].

Recently, Jain et al have developed and compared several routing algorithms in DTNs [2], including MED (Minimum Expected Delay). With MED, as proposed in [10], the routing table is recomputed each time a contact arrives and messages are exchanged if the topology suggests that a connected node is "closer" than the current node.

However, MED is a routing metric based on the contact summary, such as the average waiting time, propagation delay and transmission delay. It uses the path with the minimum average delay for all messages with the same source-destination pair. This approach depends on the average information and it doesn't take into account the dynamic changes in the network. As we'll illustrate in Section III, we need consider uncontrolled random moving characteristic of mobile devices and the practical average delay accurately. Furthermore, these routing approaches are designed either for unicast, which is to deliver a message to a determined destination, or for multicast, which is to distribute a message to a group of members. Contrarily, Anycast in DTN aims to deliver a message to any one of a group, usually the nearest one which requires a model to define the precise semantics of anycast in DTNs.

\section{EMDDA-BASED ANYCAST IN DTNS}

In this section, we first introduce our network model. Then we present the anycast semantics in DTNs. Finally, we develop our routing algorithm based on our anycast semantics. Our

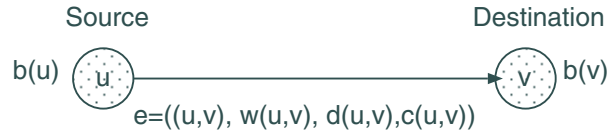

Fig. 2. An edge in a DTN graph, which is characterized by its source and destination nodes, plus a PDF of mobile device leaving $\left(w_{e}\right)$, a moving delay $\left(d_{e}\right)$ and a storage capacity $\left(c_{e}\right) . b(u)$ is the storage capacity of node $u$.

algorithm is based on the expected shortest path routing algorithm in which routing decision can be re-made at intermediate nodes according to the movement of mobile devices.

\section{A. Network Model}

In this paper, we use a different DTN model from the one in [2]. The DTN graph is a directed graph $G=(V, E)$, where $V$ is the set of nodes and $E$ is the set of edges. An edge between node $u$ and $v$ means that there exist some mobile devices moving from the initial node $u$ (source) to the terminal node $v$ (destination) (see Fig. 2). The storage capacity on all the nodes and the mobile devices is limited. We assume that every mobile device that moves between the same initial node, $u$, and terminal node, $v$, has the same moving speed, thus having the same moving delay, $d(u, v)$, from the source to the destination. The departure time of the mobile devices is a random variable and follows some given probability distribution. $w(u, v)$ is the probability distribution function of the waiting time until a mobile device leaves from the source to the destination. $c(u, v)$ is the storage capacity of the mobile device.

We assume nodes in the network are stationary and generate messages, while mobile devices' mobility can't be controlled by the nodes and do not generate messages themselves. Nodes might be disconnected themselves, thus mobile devices can act as carriers to deliver messages for the nodes. Before it departs, a mobile device's initial node uploads selected messages. Upon arriving at its terminal node, it offloads the messages.

Fig. 1 illustrates a simple example of our DTN model, in which a single node represents a cluster of users: the cluster is relatively stationary and the users only move within it. There are some mobile devices, such as shuttle buses, cars or people, moving among the clusters. The exact schedule of the moving devices is unknown, but their moving patterns can be obtained.

\section{B. Anycast Semantics}

Due to the long delivery delay in DTNs, group membership may change during the delivery of a message, introducing ambiguity in anycast semantics. The anycast routing scheme has to deal with such membership dynamics. Next we describe three anycast semantic models that allow message senders to explicitly specify the intended receivers of a message.

Current Membership Model To determine the intended receivers of a message, a key element is to explicitly identify the time interval during which the receivers are defined. In the Current Membership (CM) Model, a message should be delivered to a node which is a destination group member when the message arrives at it.

Consider an example of such a model. In a resource discovery system, some index servers, which hold the index 
information of all resource in the system, are placed in the network according to some performance objective, such as limiting the response time of resource lookup, or satisfying the traffic constraints on each edge. These index servers form an anycast group. If one of them leaves, or new members join the network, the placement of servers might change in order to meet the response time limit or traffic constraint. Therefore, the receivers of the anycast message may change over time. With the CM model, a message requesting for some resource index should be sent to any of the current index servers.

Temporal Interval Membership Model In the Temporal Interval Membership Model (TIM), a message includes an interval that specifies the period during which the group members are defined. For a message for an anycast group $G$ with temporal interval $\left[t_{1}, t_{2}\right]$, its intended receiver must be a member of group $G$ at any time during the interval.

Using the above example, in the TIM model, if the membership interval is $[0,100]$, the message must be delivered to one node which was an index server all through that period, even if when it arrives at the node, the node is not an index server anymore.

Temporal Point Membership Model In the third model, a message also includes a membership interval. For a message for an anycast group $G$ with temporal interval $\left[t_{1}, t_{2}\right]$, its intended receiver at least should be a member of group $G$ at some time during the interval.

Similarly, using the previous example with the interval $[0,100]$, any node which was an index server during that period of time is a desired destination. If the interval is set to $[0,+\infty]$, the intended receiver could be anyone of the nodes which were or are an index server since time 0 .

The above three models can be used in practical applications according to different context. In the next sections, we use the $\mathrm{CM}$ model to define our anycast routing metric and algorithm.

\section{Expected Multi-Destination Delay for Anycast}

We propose a metric EMDDA (Expected Multi-Destination Delay for Anycast) which accurately indicates the delay from a node to the nearest member of the destination anycast group. We use Practical Expected Delay (PED) to denote the delay between any two nodes which takes into account the probability of choosing each neighbor as the next node. EMDDA of a node $n$ to an anycast group $G$ is the smallest PED among the PEDs from $n$ to all members in group $G$.

Given the network graph $G=(V, E)$, and suppose a message $m$ which should be sent to any member of a group $D_{m}=\left\{d_{0}, d_{1}, \ldots, d_{L-1}\right\}, d_{i} \in V, 0 \leq i \leq L-1$, and $L$ is the size of the group. We assume that before a mobile device starts moving, all messages that it should carry can be uploaded as long as the storage of the mobile device is not full.

One simple anycast routing algorithm is to use the sum of the expected waiting time $(E(w(u, v)))$ and moving delay $(d(u, v))$ as the weight of each edge, then to calculate the shortest path to each group member and choose the one with the smallest path weight as the anycast destination. We called this Minimum Expected Delay (MED) approach. Let us use



Fig. 3. A simple example of changing route. In this graph, we assume the moving delay of each edge is identical. According to the MED approach, a message should take the path $u \rightarrow x \rightarrow d_{1}$. But when a mobile device from $u$ to $y$ is leaving, the better choice is the route $u \rightarrow y \rightarrow d_{2}$.

$M E D(s, d)$ to represent the minimum expected delay between nodes $s$ and $d,(s, d \in V)$, which can be calculated through a shortest path algorithm, such as the Dijkstra algorithm.

While a message is waiting for a mobile device to the next node on the path of the minimum expected delay, another mobile device to another destination comes, should the message change its next-step node or destination and use this mobile device? Yes, if some conditions hold. Fig. 3shows a simple example of changing route. According to the MED approach, an anycast message heading for a group $\left\{d_{1}, d_{2}\right\}$ should follow the path $u \rightarrow x \rightarrow d_{1}$. While the message is waiting a mobile device to node $x$, a mobile device to node $y$ comes, the message should change its route, take the mobile device to y and arrive at $d_{2}$ finally, because the leaving time of mobile devices is random and the expected waiting time can't accurately reflect the specific situation that each message meets on its way to its destination.

We introduce a new routing metric called Practical Expected Delay (PED) to characterize the expected delay of taking different paths with corresponding probability between a node pair. For a node $c, N b(c)$ represents the set of all its neighbors. Let $P(c, d, a)$ be the probability that a message at node $c$ to destination node $d$ takes one of node $c$ 's neighboring nodes, $a$, as the next step node, i.e. $a \in N b(c)$. Then $\operatorname{PED}(c, d)=$ $E(w(c, d))+\sum_{a \in N b(c)} P(c, d, a) \cdot(d(c, a)+P E D(a, d))$

Next, we will calculate the value of $P(c, d, a)(a \in N b(c))$. If $d(c, a)+M E D(a, d)>M E D(c, d), P(c, d, a)=0$, i.e. while waiting, even if a mobile device to node $a$ is ready to move, the message won't take it. Let set $A(c, d)=\{a \in$ $N b(c) \mid(d(c, a)+M E D(a, d)) \leq M E D(c, d)\} . P(c, d, a), a \in$ $A(c, d)$, is equal to the probability that a mobile device to node $a$ leaves earlier than any mobile device to any other node in the set $A(c, d)$.

$$
P(c, d, a)=\int_{0}^{+\infty} f_{a}(t) \cdot \prod_{b \in A(c, d), b \neq a}\left(1-F_{b}(t)\right) \cdot d t
$$

where $a \in A(c, d)$. Here $f_{a}(t)$ is the probability density function of the waiting time before a mobile device is about to leave from $c$ to $a$, and $F_{b}(t)$ is the distribution function of the waiting time that a mobile device is leaving for $b$.

Assume on edge $i$, the departure times of mobile devices follows Poisson distributions with $\lambda=\lambda_{i}, i=0,1, \cdots, p-1, p$ is the size of $N b(c)$, i.e. the number of the neighbors and the 




Fig. 4. The EMDDA-based anycast routing algorithm in DTNs.

number of the edges at node $c$. Then $E(w(c, d))=\frac{1}{\sum_{a \in A(c, d)} \lambda_{a}}$,

$$
\begin{aligned}
P(c, d, a) & =\int_{0}^{+\infty} \lambda_{a} e^{-\lambda_{a} t} \cdot \prod_{b \in A(c, d), b \neq a} e^{-\lambda_{b} t} \cdot d t \\
& =\int_{0}^{+\infty} \lambda_{a} e^{-\sum_{b \in A(c, d)} \lambda_{b} t} \cdot d t=\frac{\lambda_{a}}{\sum_{b \in A(c, d)} \lambda_{b}}
\end{aligned}
$$

We extend the metric PED to anycast routing and get a metric called Expected Multi-Destination Delay for Anycast (EMDDA). $\operatorname{EMDDA}(s, D)=$ $\min \left(P E D\left(s, d_{0}\right), P E D\left(s, d_{1}\right), \ldots, P E D\left(s, d_{L-1}\right)\right), s \in V$, and $D$ is the destination node set and $D=\left\{d_{0}, d_{1}, \ldots, d_{L-1}\right\}$.

The key advantage of EMDDA is that it can reflect the expected delay between a pair of nodes by taking all possible paths into account instead of only the shortest path.

\section{Routing algorithm}

Our anycast routing algorithm is based on the metric EMDDA, shown in Fig. 4. It can be seen that if mobile devices move infrequently between two nodes, the edge between them will have high expected delay. Thus the shortest path algorithm won't use these edges. However, if the edge is available immediately, its waiting time becomes zero at this moment and it may be a very good choice for routing. One advantage of our routing algorithm is that it can make use of this phenomenon.

\section{Performance Evaluation}

In this section, we evaluate the performance improvement EMDDA-based algorithms can achieve over MED-based algorithms when applied to anycast in DTN environments.

\section{A. Simulation Setup}

We employ Waxman Network Topology Generator [11] to generate a random graph of 100 nodes and set lambda to 0.6 ,

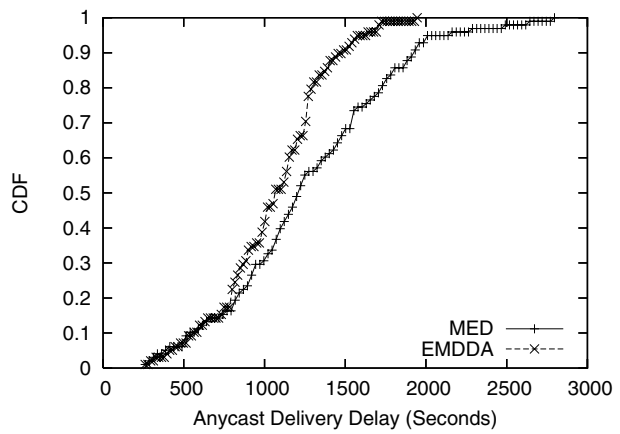

Fig. 5. CDF of anycast delivery delay (ADD). The mean message inter-arrival time is 100 seconds and the mobile device storage capacity is 300 messages.

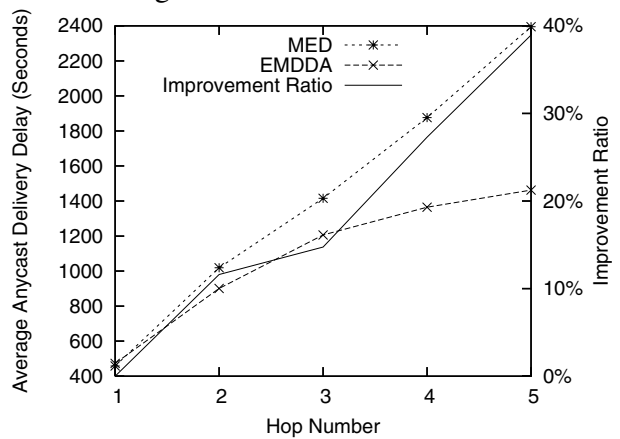

Fig. 6. Relationship of average anycast delivery delay (AADD) with hop number and improvement ratio of delay. Here, the mean message inter-arrival time is 100 seconds and the mobile device storage capacity is 300 messages.

alpha to 0.4 , beta to 0.2 . The qualitative results with other set-tings are consistent with what we present here. In extreme cases where nodes in the topology all have small node-degree (e.g. 2), the improvement of EMDDA diminishes since the routing alternatives are few.

The leaving time of mobile devices on each edge follows Poisson distributions and the mean interval time is selected randomly from 600 to 6,000 seconds. The moving delay on each edge is a number between 60 and 600 seconds, which is in proportion to the distance between the nodes. We assume that the storage capacities of mobile devices are the same and they vary from 300 to 800 messages.

Only anycast traffic is considered. For each anycast session of 200 messages, we randomly pick two nodes as the anycast destination group and one other as the anycast source. The messages are generated according to a Poisson process.

\section{B. Performance Metric}

The primary performance metric, Anycast Delivery Delay (ADD) of a message, is defined as the time to route it from its sender to any node in its anycast destination group. Average Anycast Delivering Delay (AADD) is the average ADD of all possible anycast sessions.

Average max queue length is the average of the max queue lengths on all nodes. Messages will be queued on intermediate nodes if the mobile devices to the next hop are not available immediately or they do not have sufficient capacity to carry. Messages may be dropped if the queue length exceeds the 


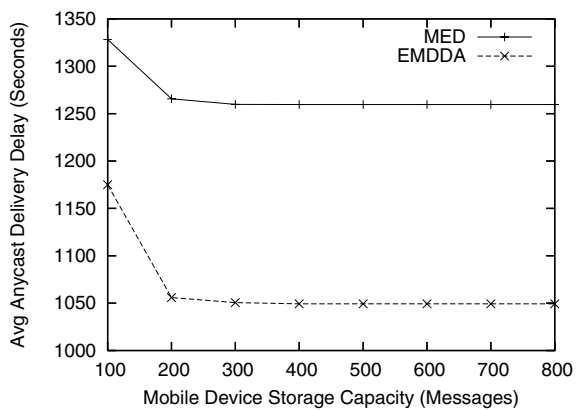

Fig. 7. Average anycast delivery delay (AADD) over mobile device storage capacity. Here the mean message inter-arrival time is 100 seconds.

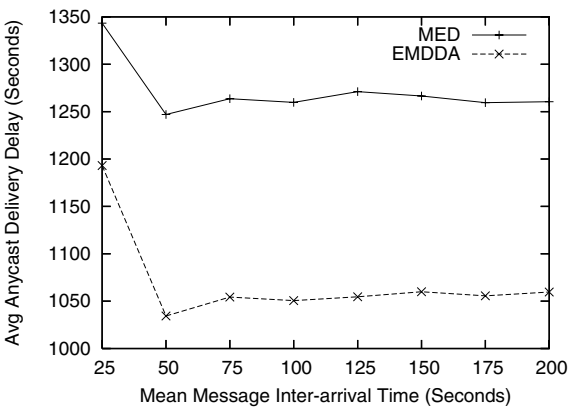

Fig. 8. Average anycast delivery delay (AADD) over mean message inter-arrival time. Here the mobile device storage capacity is 300 messages.

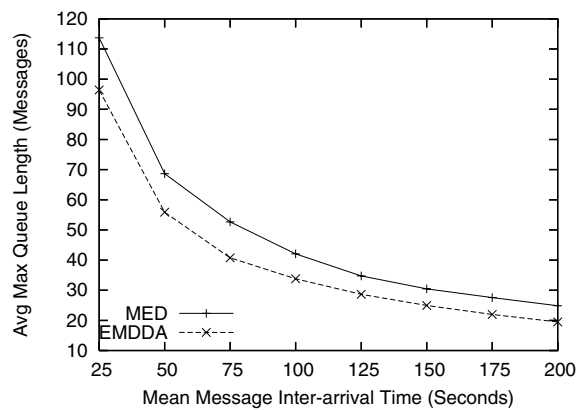

Fig. 9. Average max queue length of all nodes with mean message inter-arrival time. Here the mobile device storage is 300 messages. storage capacity allocated by intermediate nodes. This metric evaluates storage capacity required on intermediate nodes.

\section{Results}

We first compare the cumulated distribution function (CDF) of ADD between EMDDA and MED algorithms in Fig. 5. We can see that about $90 \%$ of the S-D pairs in EMDDA have ADDs below 1,500 seconds while the number for MED is only $65 \%$. EMDDA can effectively reduce the delay in anycast routing. In terms of the maximum ADD, the number for EMDDA is 1,945.2 seconds, which is $30.4 \%$ lower than that of MED, 2,795.6 seconds.

We expect that the improvement of EMDDA over MED will increase as the hop number increases since EMDDA can route messages to a closer destination intelligently along the route. In other words, more hops provide more chances for EMDDA to switch to a shorter path. Fig. 6 confirms our expectation. For one-hop routing, EMDDA and MED perform almost the same, while the improvement reaches $38.9 \%$ for five-hop routing.

In Fig. 7 and Fig. 8 we show respectively the effect of mobile device capacity and message inter-arrival time. For both EMDDA and MED, increasing storage capacity will reduce the AADD because mobile devices can carry more messages in one delivery and consequently reduce the queuing delay in intermediate nodes. Similarly, long message interarrival time will also decrease the AADD. Fig. 7 shows that EMDDA reduces the average delay by $11.3 \%$ on average.

The comparison of average max queue length (MQL) with mean message inter-arrival time is shown in Fig. 9. As the mean message inter-arrival time increases, for both MED and EMDDA, the average MQL decreases. However, the average MQL of EMDDA is always smaller than that of MED. The maximum improvement EMDDA gains over MED is $22.6 \%$ with the average improvement being $19.2 \%$. This set of simulation shows that EMDDA can effectively reduce space overhead required on the intermediate nodes.

\section{CONCLUSION}

In this paper, we analyze the anycast semantics for DTN based on a new DTN model. Three anycast semantics models are proposed, which unambiguously define the intended receivers of messages and have various applications in DTN environments. We then present a novel routing metric named EMDDA and a corresponding routing algorithm for anycast routing based on the $\mathrm{CM}$ model. The main advantage of EMDDA is that it depicts the practical expected delay for anycast more accurately than metric MED. Our simulation results show that EMDDA can reduce the average delay by $11.3 \%$ on average compared to MED and reduce the amount of buffer/space overhead by $19.2 \%$ on average.

The anycast routing algorithm proposed in this paper does not consider network traffic during its routing selection. As one of the interesting future works, we can extend our anycast routing scheme to incorporate both node storage constraint and network traffic dynamics.

\section{REFERENCES}

[1] http://www.strawberry-fair.org.uk/.

[2] S. Jain, K. Fall, and R. Patra. Routing in a Delay Tolerant Network. In Proc. of the Conference on Applications, Technologies, Architectures, and Protocols for Computer Communication, pages 145-158, Portland, OR, USA, 2004.

[3] D. Katabi and J. Wroclawski. A Framework for Scalable Global IPAnycast (GIA). In Proc. of the Conference on Applications, Technologies, Architectures, and Protocols for Computer Communication, Stockholm, Sweden, 2000.

[4] S. Bhattachargee, M. Ammar, E. Zegura, N. Shah, and Z. Fei. Application Layer Anycasting. In Proc. of the IEEE INFOCOM'97, 1997.

[5] V. D. Park and J. P. Macher. Anycast Routing for Mobile Networking. In Proc. of the IEEE Military Communications Conference, volume 1, pages $1-5,1999$.

[6] A. Vahdat and D. Becker. Epidemic Routing for Partially-connected Ad Hoc Networks. Technical report, Duke University, 2000.

[7] K. Tan, Q. Zhang, and W. Zhu. Shortest Path Routing in Partially Connected Ad Hoc Networks. In Proc. of the IEEE GLOBECOME'03, volume 2, pages 1038-1042, 2003.

[8] W. Zhao, M. Ammar, and E. Zegura. Controlling the Mobility of Multiple Data Transport Ferries in a Delay-Tolerant Network. In Proc. of the IEEE INFOCOM'05, Miami, Florida, USA, 2005.

[9] W. Zhao, M. Ammar, and E. Zegura. Multicasting in Delay Tolerant Networks: Semantic Models and Routing Algorithm. In Proc. of the 2005 ACM SIGCOMM Workshop on Delay-Tolerant Networking, Philadelphia, PA, USA, 2005.

[10] E. P. C. Jones, L. Li, and P. A. S. Ward. Practical Routing in DelayTolerant Networks. In Proc. of the 2005 ACM SIGCOMM Workshop on Delay-Tolerant Networking, Philadelphia, PA, USA, 2005.

[11] I. Kaj and R. Gaigalas. http://www.math.uu.se/ ikaj/courses/matlab/. 